\title{
Construction et importation des classements ethniques.
}

Allochtones et immigrés aux Pays-Bas et en Belgique

Dirk Jacobs et Andrea Rea

\section{(Q) OpenEdition}

\section{Journals}

Édition électronique

URL : https://journals.openedition.org/remi/2487

DOI : 10.4000/remi.2487

ISSN : $1777-5418$

Éditeur

Université de Poitiers

Édition imprimée

Date de publication : 1 juin 2005

Pagination : 35-59

ISBN : 2-911627-40-7

ISSN : 0765-0752

Référence électronique

Dirk Jacobs et Andrea Rea, "Construction et importation des classements ethniques. », Revue européenne des migrations internationales [En ligne], vol. 21 - $n^{\circ} 2$ | 2005, mis en ligne le 01 octobre 2008, consulté le 14 avril 2022. URL : http://journals.openedition.org/remi/2487 ; DOI : https://doi.org/ 10.4000/remi.2487

Ce document a été généré automatiquement le 14 avril 2022

(C) Université de Poitiers 


\title{
Construction et importation des classements ethniques.
}

\author{
Allochtones et immigrés aux Pays-Bas et en Belgique
}

Dirk Jacobs et Andrea Rea

1 Dans la plupart des Etats européens, classer et compter les nationaux et les étrangers ne souffre d'aucune illégitimité. Au contraire, cette classification constitue la manière la plus appropriée pour rendre compte de la migration ou de la circulation. La construction européenne a conduit à la formalisation d'une catégorie intermédiaire entre le national et l'étranger: le ressortissant d'un Etat de l'Union européenne. Le recours à la catégorie juridique de ressortissant plus qu'à celle, juridique et sociologique, d'étranger tend à accroître la distance entre ceux considérés comme étrangers et les autres. Le national, l'étranger, l'européen (au sens institutionnel) sont des catégories juridiques qui connaissent une traduction en catégories statistiques. Par contre, classer et compter les individus sur la base de leur origine ethnique ne bénéficie pas de la même légitimité à l'inverse de ce que l'on observe aux Etats-Unis, au Canada ou au Brésil. Dans ces pays, la différenciation ethnique et raciale participe de la vision et division du monde social. Les relations interethniques paraissent y avoir autant de consistance et de pertinence que les rapports de classes ou de genre. Les statistiques officielles enregistrent les différences ethniques et raciales qui s'appuient sur des classements institutionnels consensuels. Aux Etats-Unis, la construction statistique des groupes ethniques et raciaux n'a provoqué un large débat que lors de l'introduction de la catégorie de " mixed race » (la catégorie raciale mixte) dans le recensement de 2000 (Amaro et Zambrana, 2000 ; Krieger, 2000 ; Lee, 1993 ; Nobles, 2000 ; Riche, 1999 ; Sondik et alli, 2000 ; Waters, 2000 ; Williams et Jackson, 2000).

2 En Europe, les classements ethniques ne connaissent pas une traduction institutionnelle et statistique bien que leurs usages soient très fréquents dans la vie quotidienne et dans les interactions ordinaires. Dès lors que des discriminations ethniques et raciales sont établies, ils peuvent même être considérés comme un référentiel mobilisé par les acteurs lors de pratiques d'attribution et de distribution des bien sociaux (emploi, logement, etc.). Néanmoins, la reconnaissance de pratiques 
discriminatoires ne suffit pas à assurer le bien fondé de la construction de catégories ethniques dans les statistiques. La discordance entre l'ampleur du débat que suscite l'opportunité de la construction de catégories ethniques dans les statistiques, notamment en France (Spire et Merllié, 1998; Blum, 2002), par rapport au faible nombre de travaux scientifiques prenant pour objet les modes de constitution des classements ethniques (Bulmer, 1996; Simon, 1997, 1998; Aspinall, 2002 ; Lie, 2002) témoigne du contenu politique de la question. En effet, la passion politique qui alimente les débats et les productions scientifiques, souvent plus normatives qu'analytiques, démontrent à suffisance que la définition des catégories statistiques ethniques ne relève pas uniquement d'une question technique. La construction de ces catégorisations interroge les fondements de projets politiques, de la formation et perpétuation des Etats nations, les rapports entre l'Etat, les citoyens et les groupes sociaux.

3 L'histoire nous a montré que les statistiques ethniques peuvent être utilisées à des fins de contrôle, de stigmatisation, de ségrégation, voire d'extermination. Inversement, les groupes ethnicisés peuvent avoir intérêt face aux discriminations qu'ils subissent à ce que leur représentation statistique soit objectivée. L'adoption, en juin 2000, de la directive européenne relative à la mise en œuvre du principe d'égalité de traitement entre les personnes sans distinction, notamment de race ou d'origine ethnique, vient alimenter opportunément le débat sur la pertinence de la construction de catégories statistiques ethniques (Simon et Stavo-Debauge, 2004). En effet, les statistiques ethniques constitueraient un outil indispensable pour apprécier l'ampleur des pratiques discriminatoires et pour évaluer les effets des politiques d'égalité de chances et de lutte contre la discrimination. Un élément contribue à complexifier le débat particulièrement là où la vision et la division du monde ne s'opèrent pas légitimement selon les catégories ethniques: les projets politiques qui cherchent à restreindre des droits ou à les élargir grâce aux catégories ethniques conduisent toujours à un renforcement des classements ethniques ordinaires dans l'intelligibilité du social.

4 En matière de statistiques ethniques, deux traditions sont schématiquement présentes en Europe. En France, en Allemagne et dans les pays du sud de l'Europe, les catégories statistiques dominantes distinguent les individus sur la base de leur nationalité. La classification statistique retient principalement deux catégories: le national et l'étranger. La catégorie "étranger» renferme deux figures différentes en droit: le ressortissant d'un Etat membre de l'UE (Union Européenne) et le ressortissant d'un Etat tiers. En introduisant la catégorie statistique « population immigrée » (INSEE, 1999), la France a cherché à mesurer l'apport démographique de l'immigration sans vouloir dénombrer des groupes ethniques. Par contre, dans des pays du nord de l'Europe, des données sont produites sur le nombre de personnes qui sont des nationaux et celles qui ont une origine étrangère. En plus de la distinction entre national et étranger, le Royaume-Uni et les Pays-Bas répertorient par exemple l'ethnicité ou l'origine étrangère de ses citoyens. Aux Pays-Bas, la catégorie d'allochtone ${ }^{1}$ (allogène en français) a été instituée pour identifier l'origine ethnique ou étrangère des habitants. Cette catégorie, au départ statistique, s'est rapidement transformée en une catégorie sociale présente dans le langage ordinaire, agissant dès lors comme une catégorie ethnique ordinaire.

5 La Belgique se trouve à l'intersection de ces deux traditions. Les Francophones de Bruxelles et de Wallonie tendent à suivre la tradition française refusant la construction 
statistique de catégories ethniques alors que les Néerlandophones de Flandre et de Bruxelles ont opté pour la constitution de catégories ethniques inspirées de l'exemple hollandais en distinguant les Belges autochtones des allochtones ${ }^{2}$. En Flandre, comme aux Pays-Bas, le terme allochtone est largement utilisé dans les espaces scientifique, politique, juridique, institutionnel et médiatique pour désigner des personnes issues de l'immigration - surtout non-européenne. Cette différence de conception au sein d'un même Etat nation n'a pas encore conduit la Belgique à produire des données fédérales classant et comptant les allochtones. Cette contribution entend proposer une analyse de la constitution des catégories ethniques, et en particulier celle d'allochtone aux Pays-Bas et de son importation et de sa traduction en Belgique. Etant donné que la désignation des groupes ethniques est liée d'une part, aux catégories ordinaires et institutionnelles de perception et d'autre part, aux politiques d'intégration des immigrés et de leurs descendants, nous proposons d'inscrire l'émergence des catégories ethniques au sein de ces deux processus.

\section{La politique des minorités aux Pays-Bas}

Durant les années soixante, les Pays-Bas ont fait appel à de nombreux travailleurs immigrés pour combler la pénurie de main-d'œuvre dans certains segments du marché de l'emploi. Lors de l'arrêt de l'immigration en 1974, l'entrée d'étrangers sur le territoire s'est maintenue, bien que dans une moindre mesure, en raison du regroupement familial et de l'arrivée de demandeurs d'asile. À la même époque, un grand nombre de "nouveaux Néerlandais" originaires des anciennes colonies (Surinam, Aruba ou Antilles) gagne la métropole. La société néerlandaise prend alors conscience de l'inclusion permanente en son sein de personnes aux origines culturelles diverses. Constatant que certaines catégories d'étrangers et de Néerlandais provenant des anciennes colonies sont socialement défavorisées, les responsables politiques initient une politique active d'intégration. Le gouvernement confie à un collège d'experts, le Wetenschappelijke Raad voor het Regeringsbeleid (WRR, Conseil Scientifique pour la politique gouvernementale), la tâche de définir une politique en la matière. Le rapport du WRR (1979) intitulé Etnische minderheden (Minorités ethniques) jouit d'une grande influence auprès des responsables politiques et entraîne une modification importante de l'orientation de la politique d'intégration. Il propose la mise en œuvre d'une politique d'intégration visant à une meilleure participation des immigrés à la vie sociale et économique (Entzinger, 1993: 405). Cette politique doit d'une part, promouvoir l'inclusion des immigrés et l'égalité des chances pour la population d'origine étrangère et d'autre part, propager l'idéal d'une société multiculturelle. En 1980, le gouvernement admet officiellement que le retour d'une majorité des immigrés est improbable et lance une politique coordonnée pour les dites «minorités ethniques" ".

7 Il serait erroné de sous-estimer le poids des raisons stratégiques par rapport aux considérations de principe dans ce changement d'orientation. L'objectif du monde politique néerlandais consiste également à maîtriser le facteur «étranger » dans la société. Ainsi, l'émergence d'un consensus sur la question de l'intégration procède également des conséquences tirées des attentats terroristes perpétrés par la minorité moluquoise en 1973, population que le gouvernement a négligée pendant des années. La gestion du contentieux colonial, avec la présence aux Pays-Bas de nombreuses 
personnes venant des anciennes colonies mais disposant de la nationalité hollandaise, joue un rôle fondamental dans la manière dont le gouvernement a pensé la politique d'intégration, notamment des immigrés (Jacobs, 1998a). Enfin, les accords tacites, négociés en coulisse par les partis politiques, qui sont au fondement du consensus hollandais, cherchent à éviter la politisation de la question immigrée et à isoler, ainsi, les partis d'extrême droite.

Dans le discours gouvernemental, la notion de « minorité ethnique » ne correspond pas entièrement à celle d'immigré ou à celle de personne d'origine étrangère. Le document officiel stipule que la politique des minorités ethniques ne s'applique qu'aux groupes d'immigrés "pour la présence desquels les autorités ressentent une responsabilité spéciale (à cause du passé colonial ou parce qu'ils ont été recrutés par les autorités) et qui se trouvent en situation minoritaire " (Minderhedennota, 1983:12). La politique des minorités ethniques concerne d'une part, les Surinamiens, les Antillais, les Arubans, les Moluquois et d'autre part, les Marocains, les Turcs et les autres travailleurs immigrés de l'Europe méditerranéenne (Italiens, Espagnols, Portugais, Grecs et (ex-)Yougoslaves). Un groupe d'origine étrangère n'est considéré comme une minorité ethnique que s'il est jugé être structurellement dans une situation socioéconomique désavantageuse (Entzinger, 1993). Dès lors, les Tsiganes et les demandeurs d'asile font aussi partie des minorités ethniques alors que les autres étrangers, dont ceux provenant des pays limitrophes (Belgique et Allemagne) n'en font pas partie. La catégorie minorité ethnique cumule ainsi des critères d'extranéité et de condition sociale.

9 Les principaux axes de la politique des minorités sont la promotion du développement d'une société pluriethnique et l'émancipation des communautés ethniques, l'extension de l'égalité dans le domaine juridique et l'amélioration de la position économique et sociale des minorités. Le premier volet qui vise la promotion de la société multiculturelle met l'accent sur le groupe plutôt que sur l'individu (Entzinger, 1993 : 406). Le développement des cultures d'origine des immigrés est facilité par le soutien à la création d'organisations ethniques. Ces associations, qui organisent des activités culturelles et sportives, reçoivent souvent des subventions publiques. Des représentants des principales associations siègent dans des conseils consultatifs pour les minorités ethniques (niveau national et local) qui émettent des avis sur toute mesure politique les concernant. Cette approche de reconnaissance collective s'enracine en partie dans la structure en piliers ("verzuiling») de la société néerlandaise. Chaque groupe social et religieux dispose de ses propres associations et évolue au sein de ses propres structures (Chambon, 2002) tout en s'émancipant dans la société. Cette structure sociale donne naissance à la démocratie consociative (Lijphart, 1977) au sein de laquelle la décision politique repose avant tout sur la négociation et les compromis entre les différents segments d'essence idéologique ou philosophique. Cependant, l'existence de cette structure sociale ne constitue pas une raison suffisante pour expliquer l'émergence de cette politique d'intégration puisque la Belgique, structurée de la même manière, n'a pas totalement produit la même politique.

Le deuxième volet de la politique des minorités ethniques cherche à améliorer la position des étrangers dans le domaine juridique en assouplissant la procédure d'acquisition de la nationalité néerlandaise. Depuis 1953, les Pays-Bas connaissent le principe du double ius soli, selon lequel les personnes nées sur le territoire national d'auteurs également nés sur le territoire national, acquièrent la nationalité. Le principe 
de ius soli s'est élargi en 1984 par la possibilité donnée à la deuxième génération d'immigrés d'acquérir la nationalité néerlandaise sur simple déclaration. Tout étranger de 18 à 25 ans, né aux Pays-Bas, peut acquérir la nationalité par voie administrative. Dans le même temps, la procédure de naturalisation a subi d'importantes modifications destinées à l'accélérer et la rendre plus attractive. De plus, le gouvernement s'est efforcé de réduire les différences de droits entre les nationaux et les étrangers en octroyant à ces derniers, en 1985 , le droit de vote et d'éligibilité aux élections communales (Jacobs, 1998b).

11 Enfin, le troisième volet de la politique des minorités ethniques vise à améliorer la position économique et sociale, en la rendant comparable à celle des groupes autochtones de même niveau d'éducation. Sur ce point, le bilan est nettement moins positif comme le constate le WRR dans son deuxième rapport (1989). Au début des années quatre-vingt-dix, Han Entzinger (1993) confirme le faible progrès dans ce domaine. L'équipe de Jan-Willem Duyvendak aboutit au même constat dix ans plus tard (Rijkschroeff, Duyvendak et Pels, 2003). Toutefois, cette conclusion appelle quelques nuances. Dans le domaine du logement social, le système de distribution nondiscriminatoire a réduit les différences entre les minorités ethniques et les autochtones de même niveau socio-économique. Par contre, dans les domaines de l'éducation et de l'emploi, la situation est moins positive malgré des améliorations. Les personnes issues de l'immigration sont beaucoup plus touchées par le chômage et bénéficient plus fréquemment de l'aide sociale, en raison de leur faible niveau d'éducation et de la discrimination ethnique à l'embauche. De même, la différence reste sensible en ce qui concerne les performances scolaires. Pour certains (Koopmans, 2002), cet échec relatif démontre la faillite de la politique des minorités toute entière alors que pour d'autres (Rijkschroeff, Duyvendak et Pels, 2003; Jacobs, 2003), il témoigne des faibles contraintes imposées par les autorités pour atteindre les objectifs fixés.

Durant les années quatre-vingt-dix, un certain durcissement s'observe dans la politique des minorités. Sans abandonner cette dernière, les gouvernements successifs délaissent les discours valorisant les minorités leur préférant ceux plus expressément intégrationnistes, voire parfois assimilationnistes. Une politique d'accueil dite d'inburgering (citoyennisation) est mise en place pour les nouveaux migrants qui sont contraints de suivre des cours de langue néerlandaise, d'introduction à la société néerlandaise et des sessions d'orientation sur le marché de travail. Cependant, cette dernière politique ne s'est pas substituée pour l'heure à la première. Avec l'émergence du discours populiste de Pim Fortuyn en 2002 et le meurtre de Théo Van Gogh en 2004, le climat discursif néerlandais est néanmoins devenu, de façon spectaculaire, très hostile vis-à-vis des minorités ethniques, surtout de confession musulmane. Il n'est donc pas improbable que les Pays-Bas suppriment dans le futur leur politique des minorités à caractère multiculturaliste et instaurent une politique beaucoup plus restrictive et assimilationniste.

\section{La population allochtone aux Pays-Bas}

13 Bien que de nombreux étrangers soient devenus néerlandais ces dernières années, l'identité d'origine de ces personnes n'a pas disparu des statistiques. Les Pays-Bas n'ont marqué aucune réticence à la production de statistiques ethniques. Sans être directement à la base de sa constitution, la politique des minorités ethniques fait un 
usage de ces statistiques pour son propre développement. Les statistiques enregistrent la population étrangère sur la base de la nationalité et elles recensent la population selon l'origine ethnique. Le modèle hollandais des statistiques ethniques présente deux particularités. La première tient au critère utilisé pour produire les catégories statistiques. Contrairement au Royaume-Uni qui a recours à un système d'autoidentification pour la constitution des statistiques ethniques, les Pays-Bas ont développé un système original basé sur un critère objectif : le lieu de naissance des parents. Si la politique publique fait référence à des minorités ethniques, la statistique néerlandaise élabore une classification des origines étrangères. La deuxième particularité renvoie à la création d'une catégorie générale « allochtone » rassemblant les personnes étrangères et une partie de celles qui sont d'origine étrangère.

Bien que la notion centrale des politiques publiques soit celle de minorité ethnique, la pratique administrative a popularisé le terme "allochtone». La construction de cette catégorie résulte à l'origine d'une démarche quasi autonome de l'agence centrale de statistiques, le Centraal Bureau voor de Statistiek (CBS). Depuis 1999, le CBS définit l'allochtone comme toute personne habitant aux Pays-Bas dont au moins un des parents est né à l'étranger. Cette définition est toujours valable aujourd'hui en 2005. Le terme n'incorpore pas une distinction culturelle a priori ${ }^{3}$. Avant cette date, le CBS enregistrait déjà des personnes sous la catégorie d'allochtone et ceci systématiquement depuis $1995^{4}$ sur la base des données des administrations municipales. Pendant cette période, deux définitions étaient proposées: une large et une restreinte. Selon la définition large, les allochtones regroupent toutes les personnes habitant aux Pays-Bas qui, soit ne sont pas nées aux Pays-Bas, soit sont nées aux Pays-Bas mais dont un des parents au moins n'y est pas né. Dans une définition plus restreinte, le CBS ne compte que les personnes nées à l'étranger dont au moins un des auteurs est aussi né à l'étranger et les personnes nées aux Pays-Bas mais dont les deux parents sont nés à l'étranger. En 1998, le CBS a tendance à privilégier la définition restreinte dans ces publications avant de reprendre, l'année suivante, à nouveau la définition large sous l'insistance du gouvernement. En 1999, le CBS opte finalement pour la nouvelle définition qui est toujours en vigueur. La définition récente d'allochtone comprend donc toutes les personnes de l'ancienne définition restreinte, en y ajoutant toutes les personnes nées aux Pays-Bas, mais dont un des parents n'est pas né aux Pays-Bas (De Valk et al., 2001). Dans le rapport annuel concernant la politique des minorités ethniques de 1999, le gouvernement stipule qu'il préfère en effet garder les personnes issues de couples «mixtes » dans la définition des allochtones. L'argumentation est la suivante: «Le groupe mixte est intéressant parce qu'ils semblent réussir mieux que le groupe dont les deux parents sont nés à l'étranger $»^{5}$.

Qu'elle que soit la portée précise de la définition et de l'opérationnalisation, la catégorie allochtone est ainsi toujours plus large que celle d'étranger puisqu'elle inclut des personnes ayant obtenu la nationalité néerlandaise. Le choix du critère retenu pour établir si une personne est allochtone témoigne d'une des préoccupations du CBS. En retenant le lieu de naissance comme critère objectif plutôt que la nationalité, l'organisme cherche aussi une manière d'enregistrer les personnes de nationalité néerlandaise provenant des anciennes colonies. Enfin, les personnes de la troisième génération issues de l'immigration sont en principe automatiquement considérées comme des autochtones, du moins dans l'usage administratif du terme. Ainsi, le critère objectif du lieu de naissance est complété par celui de la génération. Le terme de 
«personnes d'origine étrangère » recouvre ainsi, la première (née en dehors des PaysBas) et la deuxième génération (née aux Pays-Bas). Un des usages sociaux de cette catégorie quasi-ethnique est de rendre visible la diversité culturelle (tableau 1), notamment dans les grandes entités urbaines, ce que ne permet pas la lecture des statistiques sur la base de la seule variable nationalité. Spatialement, les allochtones sont surtout établis dans les municipalités frontalières et dans les quatre grandes villes du pays. En 2000, Amsterdam compte $44,4 \%$ d'habitants allochtones, La Haye et Rotterdam $40 \%$ et Utrecht presque $30 \%$.

Tableau 1 : Étrangers et allochtones, Pays-Bas, 2001-2004

\begin{tabular}{|l|r|r|r|r|}
\hline & \multicolumn{1}{|c|}{2001} & \multicolumn{1}{c|}{2002} & \multicolumn{1}{c|}{2003} & \multicolumn{1}{c|}{2004} \\
\hline Population totale & 15987075 & 16105285 & 16192572 & 16258032 \\
\hline Nombre d'étrangers & 667802 & 690393 & 699954 & 702185 \\
\hline Pourcentage* d'étrangers $^{*} 4,2$ & 4870224 & 2964949 & 3038758 & 3088152 \\
\hline Nombre d'allochtones & 18,0 & 18,4 & 18,8 & 19,0 \\
\hline Pourcentage* d'allochtones & 1488960 & 1547079 & 1585927 & 1602730 \\
\hline $\begin{array}{l}\text { Nombre d'allochtones de la première } \\
\text { génération (née en dehors des Pays-Bas) }\end{array}$ & 542871 & 566165 & 588451 & 608369 \\
\hline $\begin{array}{l}\text { Nombre d'allochtones de la deuxième } \\
\text { génération (nés aux Pays-Bas) dont } \\
\text { les deux parents sont nés à l'étranger }\end{array}$ & 838393 & 851705 & 864380 & 877053 \\
\hline $\begin{array}{l}\text { Nombre d'allochtones de la deuxième } \\
\text { génération (nés aux Pays-Bas) dont } \\
\text { un seul parent est né à l'étranger }\end{array}$ & & & & \\
\hline
\end{tabular}

* Pourcentage dans la population totale

Effectifs au 1 er janvier

Source : Centraal Bureau voor de Statistiek, (http://statline.cbs.nl/statweb/)

16 La création de la catégorie d'allochtone est relativement indépendante de la construction de la politique des minorités ethniques. Il s'agit de fait de deux processus plus au moins indépendants. Pour reprendre les concepts qu'utilise Kingdon (1984) dans sa théorie de la mise à l'agenda, la production de la catégorie d'allochtone relève d'un processus bureaucratique (policy stream) proposé par une communauté de spécialistes alors que la constitution de la catégorie des minorités ethniques relève d'un processus politique (political stream). Ces deux dynamiques sont relativement indépendantes dans leur procès de production. Cependant, la matérialité de la catégorie d'allochtone s'est imposée au fur et à mesure dans tous les champs. À partir des années quatre-vingt-dix, les autorités politiques ont tendance à utiliser la catégorie " allochtone » comme un synonyme de minorités ethniques.

Dans le langage ordinaire, le terme d'allochtone connaît certainement un glissement de sens par rapport à la définition administrative initiale. Il est communément utilisé pour désigner les personnes qui sont d'origine turque, marocaine, surinamienne, antillaise ainsi que les réfugiés provenant d'Afrique, d'Asie ou d'Amérique latine. En somme, il désigne ces "Autres » à qui ne sont pas reconnus les propriétés de l'homo nationalis néerlandais-européen. Construit au départ comme une catégorie statistique descriptive par le CBS, ce terme s'est diffusé dans le langage courant et s'est transformé en une catégorie raciale-culturaliste ordinaire désignant toute personne supposée ne pas avoir une origine "occidentale». L'usage quotidien de cette catégorie se rapporte principalement aux populations visées prioritairement par le racisme européen 
(Balibar, 1992 ; Rea 1998), les descendants des immigrés les plus stigmatisés et des immigrés provenant des colonies. Les immigrés européens et leurs descendants ne sont pas repris sous ce vocable et sont dès lors autochtonisés.

Le contenu "racial-culturaliste » que comporte cette catégorie se retrouve dans la distinction statistique que le CBS opère lui-même entre les allochtones occidentaux et les allochtones non-occidentaux depuis $1999^{6}$. Font partie de la catégorie d'allochtones occidentaux, selon le CBS : «les allochtones d'origine européenne (à l'exception de la Turquie), d'origine nord-américaine, d'origine océanique, d'origine indonésienne et d'origine japonaise ». Font partie de la catégorie d'allochtones non-occidentaux, toujours selon le CBS: « les personnes issues des immigrations turque, africaine, latinoaméricaine et asiatique, à l'exception des personnes d'origine japonaise et indonésienne ». Le principe de cette subdivision des allochtones n'est donc pas seulement ethnique. Selon les propres termes du CBS, les personnes d'origine japonaise et indonésienne sont exclues de la catégorie des allochtones non-occidentaux au motif «de leur position socio-économique et culturelle $»^{7}$. La classification renoue avec les deux contenus historiques de l'idéal-type de l'immigré : l'origine ethnique et l'origine sociale inférieure.

Tableau 2 : Allochtones occidentaux et non-occidentaux, Pays-Bas, 2001-2004

\begin{tabular}{|c|c|c|c|c|}
\hline & Allochtones occidentaux & \multicolumn{2}{c|}{ Allochtones non-occidentaux } \\
\hline & Nombre & $\begin{array}{c}\text { Pourcentage de la } \\
\text { population totale }\end{array}$ & Nombre & $\begin{array}{c}\text { Pourcentage de la } \\
\text { population totale }\end{array}$ \\
\hline 2001 & 1387036 & 8,7 & 1483188 & 9,3 \\
\hline 2002 & 1406596 & 8,7 & 1558353 & 9,7 \\
\hline 2003 & 1416156 & 8,8 & 1622602 & 10,0 \\
\hline 2004 & 1419855 & 8,8 & 1668297 & 10,2 \\
\hline
\end{tabular}

Source : Centraal Bureau voor de Statistiek, (http://statline.cbs.nl/statweb/)

En assimilant automatiquement les troisièmes générations à des autochtones, la catégorie statistique d'allochtone du CBS ne fonctionne pas totalement comme une catégorie raciale. Il n'en va totalement de même dans les interactions de la vie quotidienne. D'ailleurs, bien qu'il évite scrupuleusement d'utiliser le mot «allochtone » pour désigner la troisième génération, le CBS s'efforce quand même de la cibler. En effet, depuis 2000, il dispose de chiffres concernant «la troisième génération nonoccidentale " qui comprend toute personne qui a au moins un grand-parent né à l'étranger "non-occidental», en distinguant ceux qui ont respectivement un, deux, trois ou quatre grands-parents d'origine étrangère "non-occidentale». Des chiffres spécifiques sont donnés pour les groupes d'origine marocaine, turque, surinamienne et antillaise alors que ces informations ne sont pas disponibles pour "la troisième génération occidentale ».

\section{La politique d'intégration en Belgique}

Contrairement à celle des Pays-Bas, l'histoire coloniale belge n'a pas eu d'incidence sur l'histoire de l'immigration. Peu de personnes des anciennes colonies gagnent la métropole après l'indépendance. Si les Congolais disposent du statut de sujet belge, ils ne sont pas citoyens (nationaux) au même titre que les autres Belges. Les modes 
d'attribution de la qualité de sujet belge aux Congolais ne sont pas définis par les législations valables pour la métropole mais par des dispositions d'exception visant une population confinée dans un statut hérité de l'Ancien Régime. L'immigration en Belgique se décompose en plusieurs vagues migratoires. Dans les années cinquante, les immigrés proviennent essentiellement d'Italie. Ils s'installent majoritairement dans les zones industrielles de la Wallonie et du Limbourg. Après 1964, les immigrés viennent principalement du Maroc et de Turquie. Ils s'installent davantage dans les grandes villes et, en particulier, à Bruxelles, Anvers et Gand. Jusqu'en 1974, date de l'arrêt de l'immigration, la politique d'intégration est totalement absente. Des associations d'immigrés et des organisations belges financées par l'Etat proposent des activités d'accueil (permanences juridiques et sociales). La politique d'intégration débute véritablement en 1984 avec le changement du Code de la nationalité qui institue, notamment, le double ius soli. Par la suite, la Belgique modifie à plusieurs reprises sa législation en instituant le ius soli et en assouplissant les conditions de naturalisation. L'orientation très libérale de la dernière réforme de 2000 a permis une forte augmentation du nombre de personnes ayant acquis la nationalité belge. C'est le cas de plus de la moitié de la population d'origine marocaine. La disparition des statistiques de la population issue de l'immigration est à l'origine des débats sur les statistiques ethniques. Deux autres axes composent la politique d'intégration belge : d'une part, la lutte contre le racisme et la discrimination et d'autre part, des mesures spécifiques en matière de lutte contre le chômage et contre le désavantage social. Ces deux dernières politiques ont été instituées en réaction à la montée de l'extrême droite, essentiellement en Flandre, et aux émeutes de jeunes issus de l'immigration à Bruxelles (Rea, 2001).

21 Derrière une unité de vue sur les principes généraux, des divergences opposent les Flamands et les Francophones. Lors des débats parlementaires relatifs à l'assouplissement du Code de la nationalité, ces divergences apparaissent de manière saillante. La majorité des Flamands désire maintenir parmi les critères d'attribution de la nationalité des critères dits subjectifs (par exemple, un certain degré d'intégration culturelle) ou d'ordre linguistique (par exemple, la connaissance du néerlandais sur le territoire flamand ou, comme principe plus général, d'une des langues nationales sur le territoire belge). La majorité des Francophones ne souhaite garder que des critères dits objectifs (durée de séjour légal). Cependant, cette opposition recouvre aussi un clivage politique, les partis de droite soutiennent plus la première option et ceux de gauche la seconde. Alors que les Flamands insistent sur des critères culturels, les Francophones tendent à les nier. Toutefois, certains Flamands et Francophones, essentiellement les libéraux, demandent que les requérants témoignent de leur loyauté aux lois et aux institutions du peuple belge. Le registre argumentatif des Flamands se nourrit de références à l'ethnos alors que les Francophones privilégie le demos. Sous une autre forme, cette même différenciation s'est exprimée lors de la création, en 1993, de l'institution ayant en charge la lutte contre le racisme. Les débats concernant cette proposition se concentrent sur la dénomination de l'institution. Alors que la majorité des Flamands, s'inspirant du travail de la Commission for Racial Equality au Royaume-Uni, considèrent que le Centre à créer devrait avoir pour mission de mettre en oeuvre une stratégie de promotion de l'égalité ethnique, les Francophones s'oppose à toute utilisation du terme ethnique qui supposerait que la Belgique considère légitime la constitution de ces minorités. Entre les deux positions, celle défendue par les Flamands (Centre pour l'égalité ethnique) et celle des Francophones (Centre pour l'égalité des 
chances) un compromis digne de la démocratie consociative surgit après de nombreux débats sur des propositions alternatives (Centre pour la lutte contre le racisme). L'institution est dénommée le Centre pour l'égalité des chances et la lutte contre le racisme (Rea, 1993).

\section{Un Etat et deux visions}

Une première distinction tient au mode de mise en œuvre de la politique publique. Les gouvernements flamands, contrairement aux gouvernements francophones, produisent des notes d'orientation générale définissant les priorités de la politique publique d'intégration. Du côté francophone, au-delà des textes normatifs (décrets et circulaires), c'est la consultation de l'allocations des ressources budgétaires qui permet de restituer les orientations de la politique publique. Dès lors, l'analyse des différences à partir des seuls discours politiques ne suffit pas à établir un véritable traitement différencié, la vérification de ce dernier suppose une investigation plus minutieuse bien que malaisée. Des ressemblances au niveau des pratiques et des effets des politiques publiques sont peut-être plus grandes qu'on ne le pense. Néanmoins, une différenciation dans le discours et dans les dispositifs apparait au niveau des Communautés. En Communauté française domine un discours inspiré du « républicanisme français» de l'intégration qui tend à nier les spécificités culturelles et l'origine des immigrés et de leurs descendants alors qu'en Flandre, à l'instar des PaysBas, la reconnaissance de la diversité culturelle est admise et l'existence de minorités ethniques affirmée.

Depuis 1990, la Flandre promeut une politique de reconnaissance et de soutien aux associations issues de l'immigration qui s'organisent autour d'une identité ethnique. Cette politique des immigrés (Migrantenbeleid) a pour groupe cible «toutes les personnes résidents dans le pays qui se trouvent dans une position défavorisée à cause de leur situation socio-économique faible et/ou de leur origine ethnique, qu'ils possèdent ou non la nationalité belge » (Coördinatienota Migrantenbeleid 1992). En 1996, le gouvernement flamand adopte une nouvelle terminologie, la politique des immigrés se transforme en politique des minorités (Minderhedenbeleid). Cette dernière reconnaît l'existence de groupes ethnoculturels et trois groupes cibles sont définis: les allochtones, les réfugiés et les gens du voyage (woonwagenbewoners). Cette nouvelle orientation politique est formalisée dans le décret relatif aux minorités ethniques de 1998, très largement inspiré du modèle néerlandais (Jacobs, 2001; Jacobs, 2004 ; Verlot, 2001). Bien que le gouvernement flamand s'efforce d'avoir une politique de mainstreaming, chaque domaine d'action devant prêter attention à la diversité de la population, une politique spécifique est maintenue.

Le soutien apporté aux associations ethniques témoigne de la conviction flamande selon laquelle le maintien et le développement d'une identité propre chez les descendants des immigrés stimulent leur émancipation dans la société d'installation. La Communauté flamande a jusqu'à présent voulu soutenir le développement d'organisations ethniques afin de stimuler la concertation. Reste à savoir si cela se fera encore longtemps, car, en 2005, cette politique a fait l'objet de discussions au sein du gouvernement flamand. Depuis 2004, le gouvernement flamand a également repris des Pays-Bas la politique dite d'inburgering (citoyennisation) qui impose aux nouveaux migrants non-européens de suivre des modules de formation comportant des cours de 
néerlandais, de connaissance de la société belge et des séances d'orientation sur le marché du travail.

Du côté francophone, le discours relatif aux immigrés est identique en Wallonie et à Bruxelles. Les minorités ethniques ne sont reconnues ni dans les faits ni dans les discours. La politique spécifique est relativement marginale. La politique d'intégration est davantage inscrite dans des politiques de ciblage indirect (zones d'action prioritaire, zones de discrimination positive, etc.) fondées sur des critères sociaux (pourcentage de chômeurs, de locataires, etc.) et démographiques (pourcentage d'étrangers). Bien qu'elle soit fondamentalement impropre, la dénomination dominante dans les discours ordinaire, politique et médiatique pour désigner ces " autres de l'intérieur » est celle "d'immigrés », parfois celle " de personnes d'origine étrangère ».

En termes d'action publique, la Wallonie et Bruxelles adoptent des dispositifs différents. La représentation d'un multiculturalisme en Wallonie est généralement admise alors que toute approche en termes ethniques est rejetée. Cette vision se fonde essentiellement sur l'histoire industrielle de la région. Les immigrés et leurs descendants font partie de cette histoire parce qu'ils ont été des membres de la classe ouvrière qui constitue une composante essentielle de l'identité collective wallonne. Cette représentation dominante en Wallonie tient aussi à la composition des anciens migrants, provenant majoritairement des pays de l'Europe du sud, avec lesquels les distances culturelles et religieuses sont perçues comme résorbées. La politique d'intégration wallonne se définit essentiellement sur la base de critères sociaux; elle vise à améliorer les conditions sociales des personnes issues de l'immigration. En 1996, un décret relatif à "la population étrangère ou d'origine étrangère " organise la politique publique. Bien que ce décret énonce que la politique d'intégration est fondée sur la discrimination positive pour promouvoir l'égalité des chances, ni les dispositions légales ni les projets financés ne donnent de contenu à cette notion. Des associations d'immigrés sont financées dans le cadre de cette politique, parce qu'elle proposent des activités d'insertion sociale (alphabétisation, formation professionnelle, etc.) et non parce qu'elles ont une identité ethnique. A Bruxelles, la politique publique francophone est organisée entre 1993 et 2003 par une circulaire portant sur "l'intégration et la cohabitation des communautés locales ». La nouvelle orientation politique est contenue dans le décret de 2004 portant sur la cohésion sociale. Les termes d'immigrés et personnes d'origine étrangère sont absents. Le concept de cohésion sociale est supposé recouvrir les clivages socio-économiques et culturels au sein de la ville. Plus qu'une pratique d'euphémisation, cette nouvelle législation inaugure une pratique discursive de dénégation ("faire sans dire »). Ici aussi, les objectifs sont davantage sociaux que culturels. Comme en Wallonie, l'auto-organisation des minorités ethniques n'est pas financée. Les associations des minorités ethniques reçoivent des subsides pour leur action généraliste (amélioration du niveau scolaire, formation, animations sociales et sportives, citoyenneté, etc.). Il existe un refus de soutenir des activités touchant au domaine de l'identité, du moins dans les discours. De fait, tant en Wallonie qu'à Bruxelles des animations ayant lieu pendant le Ramadan sont financées de même que des activités interculturelles. Chez les francophones, il existe un référentiel de l'intégration des immigrés importé de France, alors que la Belgique n'est ni jacobine ni laïque. Toutefois, cette importation d'un discours qui ne reçoit pas de confirmation dans la réalité exerce une pression sur les minorités ethniques pour qu'elles se conforment au modèle francophone. Dans les localités, l'intégration passe par 
l'inclusion des minorités au sein des piliers existants. L'affirmation de l'identité minoritaire n'est reconnue et acceptée que pour ceux qui font preuve d'une réussite individuelle et sont en mobilité sociale ascendante.

Tableau 3 : Typologie des politiques d'intégration des Communautés en Belgique

\begin{tabular}{|l|l|l|l|}
\hline & \multicolumn{1}{|c|}{$\begin{array}{c}\text { Politique vis à vis } \\
\text { des immigrés établis }\end{array}$} & $\begin{array}{c}\text { Politique vis à vis des } \\
\text { nouveaux migrants }\end{array}$ & \multicolumn{1}{c|}{$\begin{array}{c}\text { Univers } \\
\text { référentiel }\end{array}$} \\
\hline $\begin{array}{l}\text { Communauté } \\
\text { flam ande }\end{array}$ & $\begin{array}{l}\text { - reconnaissance de } \\
\text { l'existence de minorités } \\
\text { ethniques } \\
\text { - politique généraliste et } \\
\text { catégorielle } \\
\text { - soutien financier aux } \\
\text { associations ethnoculturelles }\end{array}$ & Programme d'inburgering & $\begin{array}{l}\text { Modèle } \\
\text { multiculturaliste } \\
\text { néerlandais }\end{array}$ \\
\hline $\begin{array}{l}\text { Communauté } \\
\text { française }\end{array}$ & $\begin{array}{l}\text { - approche individuelle } \\
\text { - politique généraliste } \\
\text { fondée sur des critères } \\
\text { sociaux } \\
\text { - politique spécifique à } \\
\text { connotation plus sociale } \\
\text { que culturelle }\end{array}$ & $\begin{array}{l}\text { Pas de politique spécifique, } \\
\text { mais actions financées }\end{array}$ & $\begin{array}{l}\text { Modèle } \\
\text { néerlandais } \\
\text { d'inburgerngèle } \\
\text { assimilationniste } \\
\text { et méritocratique } \\
\text { français }\end{array}$ \\
\hline
\end{tabular}

La divergence des paradigmes des politiques publiques et des actions financées en Flandre, en Wallonie et à Bruxelles ne doit pas cacher une certaine convergence. Les minorités ethniques ne peuvent pas avoir d'existence dans l'espace public. Leur spécificité peut être maintenue, voire soutenue comme en Flandre, dans l'espace de la société civile, mais elles ne peuvent se constituer en groupes autonomes revendiquant une légitimité à exister comme les autres piliers de la société belge. La situation en Flandre pourrait être qualifiée de multiculturalisme inégalitaire (Martiniello, 1997) et celle de la Communauté française de multiculturalisme méritocratique (Rea, 2001). Deux raisons peuvent être avancées pour comprendre les fondements de ce traitement politique différencié. Le premier, qui semble a priori relever d'un principe, la reconnaissance de la culture de l'autre, renvoie à des raisons structurelles. L'importation du modèle néerlandais chez les Flamands tient aussi à l'histoire du mouvement flamand au sein de l'histoire de la Belgique. Les Flamands majoritaires en nombre ont subi une domination culturelle francophone de plus d'un siècle inscrite dans les pratiques sociales et incorporée dans les institutions. Ne pas renier son appartenance culturelle, entretenir et défendre la culture ont été des moteurs de la constitution de l'identité flamande. On pourrait dire que par homologie structurelle, les Flamands ne peuvent imposer à leurs groupes minoritaires ce qu'ils on vécu comme groupe minorisé. Inversement, pour les Francophones, la dimension relationnelle et stratégique domine la position adoptée plus que le simple référentiel français. Dans un Etat où ils occupent une position minoritaire tout en défendant l'identité nationale, ils adoptent une stratégie visant à transformer les nouveaux Belges en Francophones et non en sous-minorités. Franciser et belgiciser ces nouveaux venus renforce leur position face aux Flamands. La configuration des deux grands groupes nationaux et leurs rapports de force expliquent, entre autres, les positions retenues par les entités fédérées en matière d'intégration des immigrés bien que cette différenciation soit également tempérée par l'appartenance politique (Martiniello et Rea, 204). 


\section{L'importation du terme allochtone et son usage en Flandre} l'intégration des immigrés. Le terme allochtone apparait pour la première fois dans le rapport du Commissariat royal à la politique des immigrés (CRPI, 1989), institution fédérale créé en 1989 et chargée de fournir des recommandations en matière d'intégration. Utilisé par des Néerlandophones, le terme se popularise en Flandre. Dans le décret de 1998, le gouvernement flamand importe la notion d'allochtone construite par le CBS en lui faisant subir trois transformations. Le critère générationnel est élargi à la troisième génération. La situation socio-économique défavorable au fondement de la catégorie néerlandaise d'allochtone non-occidental définit la catégorie générale d'allochtone. Enfin, la définition comporte une dimension plus culturaliste. La définition dans le décret de 1998 est la suivante: «Par allochtones, on entend toutes les personnes qui se trouvent légalement en Belgique et qui remplissent en même temps les conditions suivantes, quelles possèdent ou non la nationalité belge: a) au moins un de leurs parents ou grands-parents est né en dehors de la Belgique, b) ils se trouvent dans une situation défavorisée à cause de leur origine ethnique ou leur situation socio-économique faible ».

i cette définition sert à définir le public cible de la politique spécifique en Flandre, elle ne peut être utilisée dans la collecte de données statistiques. L'administration fédérale des statistiques n'opère que la distinction entre Belges et étrangers. A ce sujet, un article de la loi relative à la statistique publique stipule que l'Institut National de Statistiques (INS) n'a pas l'autorisation de produire des statistiques concernant l'origine ethnique: «En aucun cas, les investigations et études statistiques de l'Institut National de Statistiques ne peuvent concerner la vie privée, les opinions ou activités politiques, philosophiques ou religieuses, la race ou l'origine ethnique $»^{8}$. Dans une note interne, l'Institut National de Statistique commente cet article de la façon suivante: «En excluant toute investigation sur les opinions ou activités politiques, philosophiques ou religieuses de la population, la race ou l'origine ethnique et la vie sexuelle, l'article 24 quinquies permet à l'Institut National de Statistique de rester à l'abri de toute controverse. L'Institut National de Statistique doit être un organisme neutre et indépendant ainsi qu'un instrument fiable et crédible d'administration du pays » (INS, 1986). Les Francophones refusent jusqu'à présent catégoriquement tout recensement de données relatives aux origines ethniques. L'absence de consensus au niveau fédéral interdit tout changement à ce niveau de pouvoir.

Bien que les textes de la politique des minorités définissent précisément les critères de la catégorie d'allochtones, il n'existe pas d'opérationnalisation systématique de cette notion dans les pratiques administratives et statistiques en Région flamande. Le modèle du pays voisin a été copié sans en assurer sa mise en œuvre (« dire sans faire »), ce qui est critiqué par le monde académique (Verhoeven et al., 2003). Cependant, des demandes s'expriment en ce sens (Caestecker, 2001). Dans certains cas, la démarche a été entreprise mais en suivant une procédure peu fiable. Ainsi, le service flamand de placement des chômeurs (VDAB) a construit des données agglomérées relatives à la situation des autochtones et allochtones sur le marché de l'emploi. Il a retenu pour ce faire la méthode patronymique; les allochtones sont identifiés à partir de leur nom (Van der Straeten \& Jacobs, 2004c).

Revue européenne des migrations internationales, vol. 21 - n² | 2005 
31 La seule initiative systématique d'identification des allochtones en Flandre relève jusqu'à présent de la politique de l'enseignement. En 1993, la «déclaration de nondiscrimination » (non-discriminatieverklaring) visant à une meilleure répartition des élèves étrangers ou d'origine étrangère et à lutter contre les pratiques discriminatoires est adoptée. Dès lors, des dispositifs spécifiques (onderwijsvoorrangsbeleid) de discrimination positive (plus de moyens financiers) ont concerné les écoles à haute fréquentation d'élèves allochtones (Van der Straeten \& Jacobs, 2004b). Pour identifier ces écoles, un enregistrement de l'origine ethnique des élèves a été effectué systématiquement jusqu'en $2000^{\circ}$. La disponibilité de données relatives à l'origine ethnique n'affecte pas seulement l'action publique mais aussi les objets de recherche scientifique, et partant les pressions que le champ scientifique peut exercer sur le monde politique. Il semble, en effet, que l'existence de ces données ait contribué à la multiplication des recherches scientifiques en Flandre à l'inverse de la situation en Communauté française (Van der Straeten \& Jacobs, 2004b ; Florence, 2004a). Au sein de celle-ci, les recherches avancent l'existence de pratiques discriminatoires pour expliquer les moindres performances scolaires des élèves d'origine étrangère ou leurs réorientations dans les filières professionnelles sans pour autant disposer des instruments pour les établir quantitativement.

Dans l'enseignement, les données disponibles pour la région bilingue de Bruxelles permettent d'apprécier l'apport des statistiques d'origine ethnique. Le marché scolaire bruxellois est très ségrégué ethniquement. Cependant, ce constat relève plus de la connaissance pratique des acteurs que de l'objectivation scientifique. Les parents jouissent d'une liberté quasi totale dans l'inscription de leurs enfants dans les écoles. La maîtrise de la connaissance de la distribution ethnique du marché scolaire est une des dimensions des stratégies parentales. Les modes d'enregistrement des élèves sont différents dans les écoles francophones et celles néerlandophones. Comme l'indique le tableau 4, la Communauté française ne distingue les élèves que sur la base de leur nationalité. Tout observateur, même non avisé, admettra que la distribution $69 \%$ de Belges et $31 \%$ d'étrangers ne correspond pas à la représentation sociale dominante définie en termes ethniques; la part des descendants des migrants est bien plus conséquente. L'objectivation de la différenciation des établissements sur la base de l'origine ethnique permettrait d'abandonner les expressions euphémisées, et tout aussi stigmatisantes, "d'établissements difficiles" pour qualifier ceux où les groupes minorisés sont surreprésentés tout en répertoriant ceux qui devraient afficher des dispositions d'ouverture plus effectives.

Tableau 4 : Nombre d'enfants dans les écoles maternelles francophones à Bruxelles selon la nationalité, 2000-2001

\begin{tabular}{|l|r|c|c|c|c|}
\hline \multirow{2}{*}{ Réseau } & \multicolumn{2}{|c|}{ Belges } & \multicolumn{2}{c|}{ Étrangers } & \multirow{2}{*}{ Ensemble } \\
\cline { 2 - 5 } & Effectifs & $\%$ & Effectifs & $\%$ & \\
\hline Communal & 12132 & 69,0 & 5536 & 31,0 & 17668 \\
Communauté & 919 & 58,0 & 681 & 42,0 & 1600 \\
$\begin{array}{l}\text { Libre } \\
\text { (catholique) }\end{array}$ & 9755 & 71,0 & 4025 & 29,0 & 13780 \\
\hline Total & 22806 & 69,0 & 10242 & 31,0 & 33048 \\
\hline
\end{tabular}

Source : Service des Statistiques, Ministère de la Communauté française

Données fournies par Alain Dufays, directeur du Service des Statistiques, Ministère de la Communauté française. 
En comparant ces proportions avec celles affichées dans les statistiques flamandes, les statistiques francophones laissent à penser que la présence d'élèves d'origine étrangère serait moins élevée dans les écoles francophones que dans les écoles néerlandophones (cf. Tableau 5). Dans les écoles flamandes dans la Région de Bruxelles-Capitale, l'enregistrement des élèves se fait depuis 1991-1992 sur la base de leur origine culturelle, en distinguant les «familles d'origine intérieure " (binnenlands gezin) et les "familles d'origine étrangère » (buitenlands gezin). Dans cette dernière catégorie, depuis 2002-2003, on établit la distinction entre "familles occidentales» et «familles nonoccidentales ». Bien entendu, ce n'est pas la nationalité mais l'origine ethnique qui est reprise comme critère pour discerner ces «familles d'origine étrangère ». Dans les situations de famille mixte, l'origine culturelle de la mère est privilégiée. Dans le cadre de la politique flamande de discrimination positive (onderwijsvoorrangsbeleid), la récolte systématique des données repose, jusqu'en 2000, sur le critère «objectif » de lieu de naissance de la grand-mère maternelle. Après cette date, la distinction n'est plus établie de manière systématique sur ce critère; elle est aussi établie sur la base d'une attribution subjective d'une origine (hétéro-désignation) par les autorités scolaires, ce qui introduit évidemment un biais.

Tableau 5 : Nombre d'enfants dans les écoles maternelles flamandes à Bruxelles, selon l'origine, 2000-2004

\begin{tabular}{|c|c|c|c|c|}
\hline \multirow{2}{*}{ Année scolaire } & \multicolumn{2}{|c|}{ "Origine belge » } & \multicolumn{2}{c|}{ "Origine étrangère » } \\
\cline { 2 - 5 } & Effectifs & $\%$ & Effectifs & $\%$ \\
\hline $2000-2001$ & 5513 & 55,6 & 4406 & 44,4 \\
$2001-2002$ & 5251 & 52,7 & 4711 & 47,3 \\
$2002-2003$ & 5449 & 54,4 & 4567 & 45,6 \\
$2003-2004$ & 5671 & 55,1 & 4628 & 44,9 \\
\hline
\end{tabular}

Source : Vlaamse Gemeenschapscommissie, Brussels Onderwijs Punt (http://www.bop.vgc.be/)

Étant donné leur incidence sur les orientations de la politique d'éducation, les données statistiques flamandes enregistrent aussi les langues utilisées à la maison. Ils distinguent quatre catégories: les familles néerlandophones homogènes, les familles mixtes avec un parent d'origine néerlandophone, les familles francophones homogènes et les familles allophones où les deux parents ne parlent ni le français ni le néerlandais. En 2000-2001, il apparaît que les Néerlandophones étaient largement minoritaires, puisque $14,2 \%$ des familles étaient néerlandophones homogènes, $23,4 \%$ des familles mixtes avec un parent d'origine néerlandophone, $34,9 \%$ des familles francophones homogènes et $27,5 \%$ des familles allophones. L'enregistrement de ces données permet d'évaluer la proportion de Flamands fréquentant cet enseignement et, également, de réorienter la politique d'éducation par exemple la demande de voir limiter le nombre des enfants ne parlant que le français. Ces différentes approches ne sont pas sans répercussions politiques et scientifiques. L'enregistrement ethnique, du côté flamand, donne la possibilité de cibler les écoles à haute fréquentation d'élèves d'origine étrangère afin de leur donner plus de moyens dans le cadre d'une politique d'action positive (onderwijsvoorrangsbeleid). Du côté francophone, l'utilisation des données socioéconomiques agglomérées pour déterminer les écoles à discrimination positive poursuit le même objectif. Cependant, l'optique retenue par la Communauté flamande offre des instruments pour étudier les trajectoires scolaires des enfants issus de 
l'immigration, leur orientation sélective vers certaines filières et, surtout, la ségrégation scolaire. Bien sûr des études ponctuelles peuvent également fournir ces types d'information, mais celles-ci sont souvent partielles et peu comparables dans le temps. Dans d'autres domaines tels que les recherches sur le marché de l'emploi ou sur le logement, les mêmes questions se posent. Il est ainsi particulièrement difficile d'apprécier l'ethnostratification du marché de l'emploi ou l'ampleur de la discrimination à l'embauche, tant en Flandre qu'en Wallonie et à Bruxelles (Adam, 2004 ; Balancier, 2004 ; Van der Straeten \& Jacobs, 2004c). Pour cette raison, diverses administrations flamandes, dont celle en charge de la politique d'intégration, Interdepartementale Commissie Etnisch-Culturele Minderheden, insistent pour que la collecte des données relatives à la population allochtone soit entreprise. Cette demande est pratiquement inexistante au sein des institutions francophones (Florence, 2004a).

\section{Un débat qui traverse le monde scientifique}

L'importation et la traduction de la catégorie allochtone en Belgique produit également des débats au sein du champ scientifique ${ }^{10}$ (Florence, 2004b). Preuve du poids de la pensée d'Etat dans la construction des catégories scientifiques, les chercheurs flamands ont adopté la catégorie d'allochtone alors que ceux francophones la rejettent. Les rapports de recherche rédigés par des équipes universitaires des deux communautés linguistiques contiennent des modes de désignation différents : les Flamands utilisent le concept d'allochtone et les francophones lui préfèrent celui de "populations issues de l'immigration » ou de " personnes d'origine étrangère ». Il est, toutefois, significatif de relever que dans les productions scientifiques internationales écrites en anglais, la dénomination standard de "ethnic minorities» prédomine quelle que soit l'origine linguistique des chercheurs belges.

Les arguments mobilisés par les chercheurs francophones pour refuser la catégorie d'allochtone sont de trois ordres. D'abord, elle dissout la référence à la nationalité puisqu'elle réunit sous un même terme les étrangers et des nationaux d'origine étrangère, jusqu'à la troisième génération, regroupant ainsi des personnes qui ne disposent pas de droits identiques. En opérant une distinction, somme toute arbitraire, au sein des populations issues de l'immigration en fonction de la nationalité d'origine, elle donne un contenu culturaliste à sa définition. Ensuite, la construction d'une catégorie allochtone opposée à celle d'autochtone laisse entière la définition de cette dernière; comment définir un autochtone? Enfin, la construction de cette catégorie procède d'une essentialisation des groupes sociaux qui risque de renforcer la racialisation des rapports sociaux. Pour les chercheurs flamands dont une partie a substitué le terme allochtones à ceux (comme «migranten») qu'elle utilisait précédemment au moins trois raisons justifient le recours à cette catégorie. Etant donné que les personnes d'origine étrangère utilisent elles-mêmes, en Flandre, ce terme pour s'auto-désigner, les chercheurs flamands estiment que cette catégorie n'est pas de nature prescriptive, obligeant à s'en défaire. En outre, ils considèrent qu'elle n'alimente pas le racisme dans la mesure où le parti d'extrême droite, le Vlaams Belang, lui préfère le terme de vreemdelingen (étrangers). Reconnaissant l'ethnicisation de la société flamande, ils justifient l'usage de la catégorie scientifique d'allochtone déterminée par le lieu de naissance car elle permet d'une part, d'évaluer et de lutter 
contre la discrimination ethnique et raciale et d'autre part, de répondre à la demande de certains groupes minoritaires de pouvoir défendre leurs spécificités.

Toutefois, les chercheurs des deux communautés linguistiques admettent que quelle que soit la classification utilisée, la catégorie mobilisée pour différencier ethniquement implique d'une part, un jugement d'évaluation par rapport à un groupe de référence représentant la norme et d'autre part, un renforcement de la représentation de la division ethnique du monde social. Si une opposition traverse le champ scientifique en Belgique au sujet de l'opportunité et la pertinence d'utiliser la catégorie importée et traduite d'allochtone, il se dégage, néanmoins, un consensus sur la nécessité de devoir produire des statistiques rendant compte de la différenciation ethnique mais à partir des modalités en débat et qui, elles, ne font pas encore consensus.

\section{Conclusion}

L'analyse comparée des politiques d'intégration des immigrés et des modes de désignation de leurs descendants aux Pays-Bas et en Belgique révèle l'importance de la connaissance et de la reconnaissance de l'ethnicité pour la construction des catégories statistiques ethniques. La lutte entre deux modes de désignation aux Pays-Bas, les minorités ethniques et les allochtones, confirme que si une grande variété de classifications opère dans le monde social, toutes n'ont pas le même degré de formalisation. Au départ, la catégorie d'allochtone avait un objectif descriptif alors que celle de minorités ethniques, proposée par l'espace politique, avait une portée prescriptive. La classification autochtone-allochtone est devenue incontournable dès lors qu'elle procédait simultanément de la description et de la prescription. La catégorie d'allochtone s'est imposée et elle est devenue une catégorie légitime parce qu'elle a été la plus hautement formalisée. Son succès tient moins à l'identité bureaucratique du CBS qu'à la faculté de la science statistique à en faire une catégorie instituante (Desrosières, 2000). Si le pouvoir de la catégorie d'allochtone réside ainsi dans sa haute formalisation, sa faiblesse tient à sa transformation en catégorie du pouvoir qui engendre qualifications et disqualifications. La distinction allochtone occidental et allochtone non-occidental vient renforcer le processus de racialisation de la société néerlandaise tout en donnant des instruments pour apprécier les discriminations subsistantes entre les groupes sociaux de la société néerlandaise. Cependant, en devenant la catégorie de référence dans toutes les activités sociales (ordinaires, politiques, médiatiques et scientifiques), elle devient aussi suspecte et peu fiable, comme c'est également le cas en Belgique où la catégorie a été importée et transformée. La Flandre a partiellement importé la catégorie tout en n'ayant pas les moyens de son opérationnalisation statistique, du moins au niveau des institutions ayant une compétence fédérale, en raison de l'opposition des Francophones à la reconnaissance effective des minorités ethniques et de leur dénombrement statistique. En Belgique, la lutte (adhésion ou opposition) relative à la pertinence de la catégorie d'allochtone et à la légitimité de son usage s'inscrit également en partie dans la lutte entre les conceptions de la nation des deux communautés linguistiques (ethnos vs demos) et des effets propres qu'induit l'incorporation des nouveaux nationaux issus de l'immigration.

L'analyse de la construction de la catégorie d'allochtone aux Pays-Bas et son importation en Belgique démontre que les catégories statistiques et leurs usages ne 
sont pas neutres, qu'elles peuvent servir à qualifier et à disqualifier. La catégorie d'allochtone peut receler un danger dès lors que la différenciation qu'elle désigne dissimule une infériorisation. Elle peut engendrer une naturalisation de la différenciation et de la minoration en distinguant les "vrais nationaux » de ceux qui resteront "d'origine immigrée». Toute catégorisation ethnique comporte ainsi des risques de "nominalisme essentialiste" et de réification des groupes ethniques en prenant les mots pour les choses et le signifiant pour le signifié (De Rudder, $2000: 26$ ). Cependant, les catégorisations scientifiques, particulièrement leur formalisation statistique, sont aussi subordonnées aux rapports sociaux généraux. Rappelons nous ce qu'écrit Pierre Bourdieu à ce sujet : « (...) la science qui prétend proposer les critères les mieux fondés dans la réalité doit se garder d'oublier qu'elle ne fait qu'enregistrer un état de la lutte des classements, c'est-à-dire un état du rapport des forces matérielles ou symboliques entre ceux qui ont partie liée avec l'un ou l'autre mode de classement, et qui, tout comme elle, invoquent souvent l'autorité scientifique pour fonder en réalité et en raison le découpage arbitraire qu'ils entendent imposer » (Bourdieu, 1980 : 66). La double herméneutique inhérente à l'activité scientifique ne permet pas d'imaginer la constitution de catégories scientifiques autonomes. Produits d'un contexte social et politique, les catégories scientifiques ne sont pas immuables. Elles peuvent être redéfinies lorsque que le contexte change ou lorsque leur pertinence est dévoyée par des usages sociaux valorisant plus le déclassement que le classement.

Des catégories pouvant différencier des groupes sociaux et des individus, notamment sur une base ethnique, peuvent et doivent susciter des réserves. Cependant, il faut aussi être capable de nommer afin d'agir. Patrick Simon a bien formulé le dilemme qui se pose aux chercheurs et au pouvoir politique: «(...) est-il préférable de défendre une invisibilisation des différences ethniques dans l'appareil d'observation, au risque de laisser prospérer l'occultation des pratiques discriminatoires, ou bien de construire des catégories qui, par leur seule existence, peuvent éventuellement renforcer une désignation stigmatisante des populations?» (Simon, 1997 : 9). Dans le contexte post-migratoire, la catégorie juridique d'étranger ne permet plus d'appréhender l'intégration des populations d'origine étrangère. Les recherches en sciences sociales requièrent de nouvelles catégories pour pouvoir compter et classer les personnes selon leur origine ethnique afin d'examiner leur intégration et mesurer les discriminations raciales dont elles sont victimes. La classification des groupes ethniques constitue un outil nécessaire à la mise en œuvre d'une politique efficace d'égalité de chances et de lutte contre la discrimination. L'incertitude quant aux effets performatifs des catégorisations ethniques, surtout sous sa forme statistique, nous invite plus à la vigilance épistémologique qu'à la dérobade.

\section{BIBLIOGRAPHIE}

ADAM Ilke (2004), Les personnes d'origine étrangère et l'emploi aux niveaux fédéral et bruxellois, in Marleen Brans, Dirk Jacobs, Marco Martiniello, Andrea Rea, Marc Swyngedouw, Ilke Adam, Pascal Balancier, Eric Florence, Tinne Van Der Straeten, Recherche et politiques publiques: le cas de 
l'immigration en Belgique - Onderzoek en beleid: de gevalstudie van immigratie in België, Gent: Academia Press, pp. 195-242

AMARO Hortensia et ZAMBRANA Ruth (2000), Criollo, Mestizo, Mulato, LatiNegro, Indígena, White or Black? The US Hispanic/Latino Population and Multiple Responses in the 2000 Census, American Journal of Public Health, 90 (11), pp. 1724-1727.

ASPINALL Peter (2002), Collective Terminology to Describe the Minority Ethnic Population: The Persistence of Confusion and Ambiguity in Usage, Sociology, 36 (4), pp. 803-816.

BALANCIER Pascal (2004), Les personnes d'origine étrangère et l'emploi aux niveaux fédéral et bruxellois, in Marleen Brans, Dirk Jacobs, Marco Martiniello, Andrea Rea, Marc Swyngedouw, Ilke Adam, Pascal Balancier, Eric Florence, Tinne Van Der Straeten, Recherche et politiques publiques: le cas de l'immigration en Belgique - Onderzoek en beleid: de gevalstudie van immigratie in België, Gent, Academia Press, pp. 242-266.

BALIBAR Etienne (1992), Les Frontières de la démocratie, Paris, La Découverte.

BLUM Alain. (2002), Resistance to Identity Categorization in France, in David Kertzer and Dominique Arel (Eds) Census and Ethnicity:thePolitics of Race, Ethnicity and Language in National Censuses, Cambridge, Cambridge University Press, pp. 121-147.

BOURDIEU Pierre (1980) L'identité et la représentation, Actes de la recherche en sciences sociales, $\mathrm{n}^{\circ}$ 35, 1980, pp. 63-72.

BRANS Marleen, JACOBS Dirk, MARTINIELLO Marco, REA Andrea, SWYNGEDOUW Marc, ADAM Ilke, BALANCIER Pascal, FLORENCE Eric, VAN DER STRAETEN Tinne (2004), Recherche et politiques publiques: le cas de l'immigration en Belgique - Onderzoek en beleid: de gevalstudie van immigratie in België, Gent, Academia Press.

BULMER Martin (1996), The ethnic group question in the 1991 Census of population, in David Coleman and John Salt. (Eds), Ethnicity in the 1991 Census, Volume One: Demographic Characteristics of the Ethnic, Minority Populations, London, HMSO, pp. 33-62.

CAESTECKER Frank (2001) Voorstellen tot registratie van allochtonen. Onderzoek in opdracht van de dienst Emancipatiezaken van het ministerie van de Vlaamse Gemeenschap, Brussel, EHSAL, 44 p.

CHAMBON Laurent (2002), Le sel de la démocratie. L'accès des minorités au pouvoir en France et aux Pays-Bas, Amsterdam, Universiteit Amsterdam, 255 p.

CRPI (1989), L'intégration : une politique de longue haleine, volume 1, Bruxelles, Inbel.

DE RUDDER Véronique, POIRET Christian, ET VOURC'H François (2000), L’inégalité raciste. L'universalité républicaine à l'épreuve, Paris, PUF.

DE VALK Helga, ESVELDT Ingrit, HENKENS Kène \& LIEFBOER Aat (2001) Oude en nieuwe allochtonen in Nederland. Een demografisch profiel. Den Haag, Wetenschappelijke Raad voor het Regeringsbeleid, $178 \mathrm{p}$.

DESROSIERES Alain (2000), La politique des grands nombres. Histoire de la raison statistique, Paris, La Découverte/Poche.

ENTZINGER Han (1993), L'immigration aux Pays-Bas : du pluriculturalisme à l'intégration, in Michel Wieviorka (ed.), Racisme et modernité. Paris, Editions La Découverte, pp. 400-415.

FLORENCE Eric (2004a), L'enseignement en rapport avec les populations d'origine étrangère en Communauté française de Belgique, in Marleen Brans, Dirk Jacobs, Marco Martiniello, Andrea Rea, Marc Swyngedouw, Ilke Adam, Pascal Balancier, Eric Florence, Tinne Van Der Straeten, 
Recherche et politiques publiques: le cas de l'immigration en Belgique - Onderzoek en beleid: de gevalstudie van immigratie in België, Gent, Academia Press, pp. 121-194.

FLORENCE Eric (2004b), Catégorisations et sciences sociales dans le domaine des études migratoires et ethniques », in Marleen Brans, Dirk Jacobs, Marco Martiniello, Andrea Rea, Marc Swyngedouw, Ilke Adam, Pascal Balancier, Eric Florence, Tinne Van Der Straeten, Recherche et politiques publiques: le cas de l'immigration en Belgique - Onderzoek en beleid: de gevalstudie van immigratie in België, Gent, Academia Press, pp. 319-338.

INSEE (1999), Recensement de la population, mars.

INSTITUT NATIONAL DE STATISTIQUE (1986) Loi du 4 Juillet 1962 relative à la statistique publique, modifiée par la loi du $1^{\text {er }}$ Août 1985, Bruxelles, Ministère des affaires économiques, 25 p.

JACOBS Dirk (1998a) Nieuwkomers in de politiek. Het parlementaire debat omtrent kiesrecht voor vreemdelingen in Nederland en België (1970-1997), Gent, Academia Press, 311 p.

JACOBS Dirk (1998b), Discourse, politics and Policy. The debate over voting rights for foreign residents in The Netherlands (1970-1996), International Migration Review, 32 (2), pp. 350-373.

JACOBS Dirk (2001), Immigrants in a Multinational Political Sphere: The Case of Brussels, in Alisdair Rogers \& Jean Tillie (Eds.), Multicultural Policies and Modes of Citizenship in European Cities, Aldershot, Ashgate, pp. 107-122

JACOBS Dirk (2002), L'accès à la nationalité néerlandaise de la population d'origine étrangère aux Pays-Bas, Migrations Société, 14 (80), pp. 103-117.

JACOBS Dirk (2003), Bronnenonderzoek integratiebeleid. Moet integratiebeleid mislukken?, Tijdschrift voor de Sociale Sector, 57 (12), pp. 45-47.

JACOBS, Dirk (2004), Alive and Kicking? Multiculturalism in Flanders, International Journal on Multicultural Societies, 6 (2), pp. 189-208.

JENKINS Richard (1994), Rethinking ethnicity: identity, categorization and power, Ethnic and Racial Studies, 17 (2), pp. 197-223.

KINGDON John (1984), Agendas, Alternatives and Public Policies, Boston, Little Brown.

KOOPMANS Ruud (2002), Zachte heelmeesters... Een vergelijking van de resultaten van het Nederlandse en Duitse integratiebeleid en wat de WRR daaruit niet concludeert, Migrantenstudies, 18 (2), pp. 87-92.

KRIEGER Nancy (2000), Counting accountably: Implications of the New Approaches to Classifying Race/Ethnicity in the 2000 Census, American Journal of Public Health, 90 (11), pp. 1687-1689.

LEE Sharon. (1993), Racial classifications in the US census: 1890-1990, Ethnic and Racial Studies, 16 (1), pp. 75-94.

LIE Einar (2002), Numbering the nationalities: ethnic minorities in Norwegian population censuses 1845-1930, Ethnic and Racial Studies, 25 (5), pp. 802-822.

LIJPHART Arend (1977), Democracy in plural societies: a comparative exploration, New Haven, Yale University Press.

MARTINIELLO Marco (1997), Sortir des ghettos culturels, Presses de Sciences-po, Paris.

MARTINIELLO Marco et REA Andrea (2004), Piliers, minorités ethniques et pluralisme en Belgique, in Marco Martiniello et Andrea Rea (Eds), Affirmative action, Des discours, des politiques et des pratiques, Louvain-la-Neuve/Liège, Academia, pp. 253-281.

MINDERHEDENNOTA (1983), Tweede Kamer, zitting 1982-1983, 16102 No 21. Den Haag. 
NOBLES Melissa (2000), History Counts: A Comparative Analysis of Racial/Color Categorization in US and Brazilian Censuses, American Journal of Public Health, 90 (11), pp. 1738-1745.

PRINS Baukje (2000), Voorbij de onschuld. Het debat over de multiculturele samenleving. Amsterdam, Van Gennep.

REA Andrea (1993), « Du Commissariat à la politique des immigrés au Centre pour l'égalité des chances et la lutte contre le racisme ", L'Année sociale. 1992, 1993, pp. 276-282. (http:// www.ulb.ac.be/is/revas.html)

REA Andrea (1998), « Le racisme européen ou la fabrication du sous-blanc », in Andrea Rea. (éd.), Immigration et racisme en Europe, Bruxelles, Editions Complexes, pp. 167-201. http:// www.ulb.ac.be/is/revas.html

REA Andrea (2001), Jeunes immigrés dans la Cité, Protestation collective, acteurs locaux et politiques publiques, Bruxelles, Labor.

RICHE Martha (1999), Cultural and Political Dimensions of the US Census. Past and present, American Behavioural Scientist, 42 (6), pp. 933-945.

RIJKSCHROEFF Rally, DUYVENDAK Jan-Willem \& PELS Trees (2003), Bronnenonderzoek integratiebeleid. Utrecht, Verwey-Jonker Instituut.

SIMON Patrick (1997), La représentation statistique de l'immigration. Peut-on comptabiliser l'ethnicité ?, in Jean-Louis Rallu., Youssef Courbage \& Victor Piché (Eds.), Old and new minorities/ Anciennes et nouvelles minorités, pp. 1-30, Paris, INED.

SIMON Patrick (1998), Nationalité et origine dans la statistique française. Les catégories ambiguës, Population, 3, pp. 541-568.

SIMON Patrick et STAVO-DEBAUGE Joan (2004), Les politiques anti-discrimination et les statistiques : paramètres d'une incohérence, Sociétés Contemporaines, 53/2004, pp. 57-84.

SONDIK Edward, LUCAS Jacqueline, MADANS Jennifer \& SMITH Sandra (2000), Race/Ethnicity and the 2000 Census : Implications for Public Health, American Journal of Public Health, 90 (11), pp. 1709-1713.

SPIRE Alexis et MERLLIE Dominique (1998), La question des origines dans les statistiques en France. Les enjeux d'une controverse, Le Mouvement social, ${ }^{\circ}{ }^{\circ} 188$, pp. 119-129.

VAN DER STRAETEN Tinne \& JACOBS Dirk (2004a), Het Vlaamse minderhedenbeleid en het onderwijs van kinderen en jongeren van buitenlandse origine in de Vlaamse Gemeenschap. Een blik op beleid en onderzoek, in Marleen Brans, Dirk Jacobs, Marco Martiniello, Andrea Rea, Marc Swyngedouw, Ilke Adam, Pascal Balancier, Eric Florence, Tinne Van Der Straeten, Recherche et politiques publiques: le cas de l'immigration en Belgique - Onderzoek en beleid: de gevalstudie van immigratie in België, Gent, Academia Press, pp. 77-101.

VAN DER STRAETEN Tinne \& JACOBS Dirk (2004b), De wisselwerking tussen onderzoek en beleid aangaande allochtonen in het Vlaamse onderwijs nader bekeken, in Marleen Brans, Dirk Jacobs, Marco Martiniello, Andrea Rea, Marc Swyngedouw, Ilke Adam, Pascal Balancier, Eric Florence, Tinne Van Der Straeten, Recherche et politiques publiques: le cas de l'immigration en Belgique Onderzoek en beleid: de gevalstudie van immigratie in België, Gent, Academia Press, pp. 101-121.

VAN DER STRAETEN Tinne \& JACOBS Dirk (2004c), Vlaams beleid en onderzoek aangaande participatie van allochtonen op de arbeidsmarkt. Een verkenning, in Marleen Brans, Dirk Jacobs, Marco Martiniello, Andrea Rea, Marc Swyngedouw, Ilke Adam, Pascal Balancier, Eric Florence, Tinne Van Der Straeten, Recherche et politiques publiques: le cas de l'immigration en Belgique Onderzoek en beleid: de gevalstudie van immigratie in België, Gent, Academia Press, pp. 267-285. 
VERHOEVEN Hans, ANTHIERENS Jan, NEUDT Danny \& MARTENS Albert (2003), Het Vlaams minderhedenbeleid gewikt en gewogen. Evaluatie van het Vlaams minderhedenbeleid (1996-2002). Rapport voor de Vlaamse Regering, Leuven, KULeuven.

VERLOT Marc (2001), Werken aan integratie. Het minderheden- en het onderwijsbeleid in de Franse en Vlaamse Gemeenschap, Leuven, Acco.

WATERS Mary (2000), Immigration, Intermarriage, and the Challenges of Measuring Racial/ Ethnic Identities, American Journal of Public Health, 90 (11), pp. 1735-1737.

WILLIAMS David \& JACKSON James (2000), Race/Ethnicity and the 2000 Census: Recommendations for African Americans and Other Black Populations in the United States, American Journal of Public Health, 90 (11), pp. 1728-1730.

\section{NOTES}

1. Selon la philosophe Baukje Prins (2000) le mot «allochtone» (allochtoon en néerlandais) était popularisée par la célèbre sociologue et politicienne néerlandaise Hilda Verwey-Jonker. L'opposé d'allochtone est « autochtone » (« habitant de souche »).

2. La Belgique est un état fédéral composé de trois entités fédérées reposant sur une base territoriale: la Wallonie (majoritairement francophone), la Flandre (majoritairement néerlandophone) et Bruxelles (région bilingue). Par ailleurs, il existe aussi des entités politiques dénommées les Communautés qui se définissent par la langue (néerlandais, français et allemand). Ainsi, la Communauté française est compétente, par exemple en matière d'éducation pour les écoles francophones en Wallonie et à Bruxelles, la Communauté flamande pour les écoles néerlandophones en Flandre et à Bruxelles. La Communauté germanophone gère ces matières dans les communes de langue allemande de la Belgique.

3. D'ailleurs, selon cette définition, la plupart des membres de la famille royale néerlandaise sont allochtones.

4. Les chiffres sont disponibles sur base d'estimations depuis 1972 et sur base des données auprès des administrations municipales depuis 1995 (après l'introduction du registre Gemeentelijke Basisadministratie en 1994).

5. Tweede Kamer, 1999-2000, Document 26815, p. 5, note 2.

6. Cette distinction est disponible dans les tableaux statistiques à partir des données pour 1996.

7. http://statline.cbs.nl/statweb/.

8. Article 24 quinquies de la loi du 4 Juillet 1962 relative à la statistique publique, modifiée par la loi du $1^{\text {er }}$ Août 1985.

9. On récolte des données concernant l'origine ethnique afin de dénombrer les élèves ciblés par la politique publique. Les élèves ciblés («doelgroepleerlingen ») sont des élèves dont la grandmère maternelle n'est pas née en Belgique et ne possède pas la nationalité belge ni la nationalité néerlandaise et dont la mère n'a pas continué ses études après l'âge de 18 ans. Depuis 2000, une autre définition est utilisée et l'origine ethnique n'est plus directement prise en compte pour établir les écoles bénéficiaires du système de discrimination positive. Les critères sont désormais des indicateurs d'inégalité de chances généraux (pour le débat sur l'opérationnalisation : voir Van der Straeten \& Jacobs, 2004a ; 2004b).

10. $\mathrm{Y}$ compris entre les auteurs de cette contribution. 


\section{RÉSUMÉS}

L'article propose une analyse comparée de l'émergence de l'ethnicité et de sa reconnaissance dans la construction des catégories statistiques aux Pays-Bas et en Belgique. Il interroge les conditions d'apparition de la catégorie statistique "allochtones » aux Pays-Bas. Cette dernière devait permettre de distinguer l'origine ethnique des nationaux, en identifiant les anciens colonisés devenus néerlandais. Poursuivant au départ une objectif descriptif, cette catégorie a supplanté celle de «minorités ethniques» figurant dans les discours et politiques publiques. L'analyse soutient que la classification autochtones-allochtones est devenue indispensable d'une part, parce qu'elle procédait simultanément de la description et de la prescription et d'autre part, parce qu'elle disposait d'une plus haute formalisation statistique.

La catégorie "allochtones" a été importée en Belgique où elle a subi quelques transformations. La Flandre utilise cette catégorie pour désigner l'altérité ethnique mais elle ne dispose pas des moyens de son opérationnalisation statistique en raison de l'opposition des Francophones à la reconnaissance effective des minorités ethniques et à leur dénombrement statistique. En Belgique, l'usage de cette catégorie est l'objet de luttes dans lesquelles s'opposent des conceptions différenciées de la nation des deux communautés linguistiques (ethnos vs demos).

The Construction and Import of Ethnic Categories.The Concept of "Allochthonous" in the Netherlands and Belgium.

This article presents a comparative analysis of the rise of the notion of ethnicity and its construction in statistical categories in the Netherlands and in Belgium. We look into how the statistical category of "allochthone" has developed in the Netherlands. This category should allow to trace the ethnic origin of citizens and in particular to identify the former colonial subjects who have Dutch citizenship. While the original use was purely descriptive, over time the category has come to replace the notion of "ethnic minorities" in public discourse and policy usage. Gradually the distinction between "allochthones" and "autochtones" has become indispensable in Dutch policy discourse, both because it has a descriptive and a prescriptive dimension as because it allows for a high degree of statistical formalisation.

Belgium has partially imported the notion of "allochthone", with a number of modifications. Flanders uses the category to highlight ethnic alterity but at the same time does not dispose of sufficient possibilities for adequate statistical operationalisation because the Francophones have traditionally resisted the effective recognition of the existence of ethnic minorities and has always hindered the statistical tracing of the concerned groups on the federal level. As such, the use of the notion in Belgium has even become one of the stakes of a struggle between two opposed and competing visions of the two large linguistic communities with regard to the nation (ethnos versus demos).

Construcción e importación de las categorías étnicas. Alóctonos e inmigrantes en los Países Bajos y en Bélgica.

Este artículo propone una análisis comparada del surgimiento de la etnicidad y de su reconocimiento para la construcción de categorías estadísticas en los Países Bajos y en Bélgica. Estudia las condiciones de la aparición de la categoría estadística de de "alóctono" en los Países Bajos. Debía permitir definir la origen étnica de los neerlandeses, identificando a los excolonizados que tienen la nacionalidad neerlandesa. La creación de esta categoría tenía como objetivo la descripción de la población, pero rápidamente suplantó en los discursos y las políticas públicas la de "minorías étnicas". Gradualmente la diferenciación entre "autóctonos" y "alóctonos" se reveló imprescindible porque permite simultáneamente la descripción y la prescripción, y también porque dispone de un alto grado de formalización estadística. La 
categoría "alóctonos" fue importada en Bélgica con ciertas modificaciones. Flandes la utiliza para designar la alteridad étnica, pero no dispone de datos suficientes para establecer estadísticas operacionales porque los Francófonos se niegan tradicionalmente a reconocer a las minorías étnicas y se oponen a la producción de estadísticas federales relativas a estas. En Bélgica el usa de la categoría "alóctonos" es objeto de debates que corresponden a las concepciones diferentes de la nación entre las dos comunidades lingüísticas (ethnos versus demos)

\section{INDEX}

Index géographique : Belgique, Pays-Bas

Mots-clés : ethnicité, minorités, identités ethniques

\section{AUTEURS}

\section{DIRK JACOBS}

Chargé de cours en sociologie à l'Université Libre de Bruxelles, membre du GERME (Groupe d'étude sur l'Ethnicité, le Racisme, les Migrations et l'Exclusion), Institut de sociologie, 44 avenue Jeanne, 1050 Bruxelles, dijacobs@ulb.ac.be

\section{ANDREA REA}

Chargé de cours en sociologie à l'Université Libre de Bruxelles, directeur du GERME (Groupe d'étude sur l'Ethnicité, le Racisme, les Migrations et l'Exclusion), Institut de sociologie, 44 avenue Jeanne, 1050 Bruxelles, area@ulb.ac.be, http://www.ulb.ac.be/socio/germe. 